\title{
Estudo comparativo da qualidade de vida de idosos asilados e frequentadores do centro dia
}

\author{
Comparative study of quality of life of elderly nursing home \\ residents and those attneding a day Center
}

Sandra Fiorelli de Almeida Penteado Simeão ${ }^{1}$

Géssika Araujo de Lima Martins ${ }^{2}$

Marcia Aparecida Nuevo Gatti ${ }^{1}$

Marta Helena Souza De Conti ${ }^{1}$

Alberto De Vitta ${ }^{1}$

Sara Nader Marta ${ }^{1}$

${ }^{1}$ Pró-Reitoria de Pesquisa e Pós-Graduação, Universidade Sagrado Coração. R. Irmã Arminda 10-50, Jardim Brasil. 17011160 Bauru SP Brasil. ssimeao@usc.br ${ }^{2}$ Hospital Estadual Bauru. Bauru SP Brasil.

\begin{abstract}
The aim of this study was to compare the QOL of the elderly living in nursing homes and those who attend the Day Center (Centro Dia) at the the Asilo Vila Vicentina in the city of Bauru/ SP. The sample consisted of 48 subjects, 21 men, 5 from the Day Center and 16 nursing home residents, and 27 women, 16 from the Day Center and 11 nursing homes residents, who answered the following questionnaires: socio-demographic characteristics, WHOQOL-Old, and WHOQOL-Bref. The responses were submitted to descriptive and inferential statistics to compare the QOL scores of the nursing home residents with the elderly who attend the Day Center using the Mann Whitney test. The results showed better QOL scores for the elderly who attend the Day Center, in which women stood out. Among the institutionalized elderly, women presented the worst $Q O L$ values, particularly in the Physical and Psychological domains. The domains with the lowest scores were Environment (42.6 \pm 10.7 for women in nursing homes and $44.4 \pm 9.7$ for men at the Day Center) and Intimacy (13.1 \pm 17.3 for women in nursing homes and $9.4 \pm 22.7$ for men in nursing homes). The domains with the highest scores were Social Affairs (74.0 \pm 13.6 for women at the Day Center and $68.3 \pm 10.9$ for men at the Day Center) and Death/Dying (83.6 \pm 22.0 for women at the Day Center and 80.0 \pm 32.6 for men at the Day Center).
\end{abstract}

Key words Quality of life, Health of Institutionalized elderly, Homes for the aged
Resumo Objetivou-se comparar a $\mathrm{QV}$ de idosos asilados e frequentadores do Centro Dia do Asilo Vila Vicentina, no município de Bauru/SP. A amostra contou com 48 sujeitos, sendo 21 homens, 5 do Centro Dia e 16 asilados, e 27 mulheres, 16 do Centro Dia e 11 asiladas, que responderam aos questionários: caracterização sociodemográfica, WHOQOL-Old e WHOQOL-Bref. As respostas, submetidas à estatística descritiva e inferencial, permitiram comparar os escores de QV dos asilados aos dos frequentadores do Centro Dia, por meio do Teste de Mann Whitney. Os resultados apontaram melhores escores de QV para os idosos do Centro Dia, destacando-se as mulheres. As institucionalizadas tiveram os piores valores de QV, sobressaindo-se os domínios Físico e Psicológico. Os dominios com escores mais baixos foram: Meio Ambiente (42,6 \pm 10,7 para as asiladas e 44,4 \pm 9,7 para os homens do Centro Dia) e Intimidade (13,1 $\pm 17,3$ para as asiladas e 9,4 $\pm 22,7$ para os asilados). Os mais altos foram: Relações Sociais (74,0 $\pm 13,6$ para as mulheres do Centro Dia e 68,3 $\pm 10,9$ para os homens do Centro Dia) $e$ Morte/Morrer (83,6 \pm 22,0 para as mulheres do Centro Dia e 80,0 \pm 32,6 para os homens do Centro Dia).

Palavras-chave Qualidade de vida, Saúde do idoso Institucionalizado, Instituição de longa permanência para idosos 


\section{Introdução}

A Organização Pan-Americana da Saúde (OPAS) considera o envelhecimento "um processo sequencial, individual, acumulativo, irreversível, universal, não patológico, de deterioração de um organismo maduro, próprio a todos os membros de uma espécie, de maneira que o tempo o torne menos capaz de fazer frente ao estresse do meio ambiente e, portanto, passível da possibilidade de morte"1. Segundo o Instituto Brasileiro de Geografia e Estatística (IBGE), a população de mais de 60 anos no Brasil, que era de 14,9 milhões (7,4\% do total), em 2013, deve passar para 58,4 milhões (26,7\% do total), em 2060, com aumento na expectativa média de vida do brasileiro de 75 para 81 anos $^{2}$. Mundialmente, pessoas desta faixa etária, serão em torno de dois bilhões e a maioria delas vivendo em países em desenvolvimento ${ }^{3}$.

Frente a esse processo de aumento da sobrevida da população, destaca-se a importância de garantir aos idosos não apenas maior longevidade, mas felicidade, satisfação pessoal e qualidade de vida $(\mathrm{QV})$. A Organização Mundial de Saúde (OMS) define QV como "a percepção do indivíduo de sua posição na vida no contexto da cultura e sistema de valores nos quais ele vive e em relação aos seus objetivos, expectativas, padrões e preocupações"4.

A QV é um fenômeno complexo, subjetivo e com múltiplos aspectos, sendo, portanto, de difícil interpretação. Envolve o julgamento individual de alguns domínios específicos da vida como autoestima e bem-estar pessoal, abrangendo aspectos relacionados à capacidade funcional, ao nível socioeconômico, ao estado emocional, à interação social, à atividade intelectual, ao autocuidado, ao suporte familiar, à saúde, à função sexual, aos valores culturais, éticos e religiosos ${ }^{5}$, ao estilo de vida, à satisfação com o emprego e/ou atividades diárias e ao ambiente em que se vive ${ }^{6-8}$.

O convívio com os familiares é essencial para a manutenção da QV do idoso, pois lhes traz segurança e sentimento de amor/reconhecimento. Por outro lado, pode ser altamente prejudicial quando a família não possui recursos e paciência para lidar com a consequência da senilidade e senescência, afetando negativamente sua QV. Dessa maneira, se o idoso não tem vínculo familiar ou não tem condições para contratar os serviços de um cuidador particular, a internação em uma Instituição de Longa Permanência para Idosos - ILPI, acaba sendo a única opção, fazendo com que se torne, total ou parcialmente, dependente dos cuidados oferecidos pelos cuidadores da Instituição $0^{9-11}$.

Mesmo quando a família existe e acolhe o idoso, é indispensável que o mesmo participe de atividades que o mantenham física e psiquicamente ativo, visando ao aumento da qualidade e expectativa de vida, assim como o controle da solidão. Dessa maneira, como uma modalidade de serviço de proteção social, foram criados os Centros Dia de Atenção à Pessoa Idosa ou Centros de Convivência, que oferecem atendimento multiprofissional diurno aos idosos, com ações de promoção e proteção à saúde e socialização de seus frequentadores ${ }^{12-15}$.

Ao se avaliar a QV do idoso asilado, alguns estudos evidenciam aspectos positivos da institucionalização, como adesão à farmacoterapia ${ }^{16}$, melhora no convívio social ${ }^{17}$, amenização de sintomas depressivos ${ }^{18}$, participação em atividades de lazer beneficiando a locomoção ${ }^{19}$. Por outro lado, outras pesquisas apontam uma menor QV da pessoa idosa acolhida na ILPI, destacando-se a insatisfação quanto à capacidade de tomar decisões $^{20}$, vida monótona ${ }^{21}$, perda de autonomia física e mental ${ }^{22}$, desvios nutricionais, tanto para magreza, como para excesso de peso ${ }^{23}$.

Pesquisas que compararam a QV dos institucionalizados e não institucionalizados revelam que os asilados têm uma pior percepção da sua $\mathrm{QV}$, possivelmente devido a fatores que causam a institucionalização e influenciam o julgamento, tais como idade, sexo, educação, estilo de vida, autonomia e participação social. Entre os domínios avaliados nos questionários de QV a autonomia e aspectos ambientais normalmente são os que menos satisfazem os idosos que moram nas ILPI ${ }^{14,19,24,25}$.

A QV na velhice não é um atributo do indivíduo, biológico ou psicológico, nem uma responsabilidade individual, mas um produto da interação entre pessoas vivendo numa sociedade em transformação. Assim, investigar a QV de moradores de ILSP e frequentadores do Centro Dia é imprescindível para constatar os impactos do asilamento na vida de alguns, assim como a frequência diária a um centro de atividades específico para idosos para outros, visando despertar na sociedade a necessidade de preparar o país para o cuidado mais humanizado de seus anciãos.

Dessa maneira, objetivou-se comparar a qualidade de vida de idosos asilados e frequentadores do Centro Dia do Asilo Vila Vicentina, no município de Bauru/SP. 
Estudo transversal de natureza quantitativa desenvolvido na ILPI Vila Vicentina Abrigo para Idosos de Bauru/SP, após autorização da administração da mesma, assim como do Comitê de Ética e Pesquisa com Seres Humanos da Universidade do Sagrado Coração. Foram incluídos os idosos moradores do Asilo e os frequentadores do Centro Dia. As entrevistas ocorreram nas dependências da Instituição no período de novembro de 2013 a março de 2014. Para a realização das mesmas os idosos foram esclarecidos sobre os objetivos da pesquisa, orientados para responderem aos questionários e, aqueles que concordaram, assinaram o Termo de Consentimento Livre e Esclarecido.

A Vila Vicentina de Bauru ${ }^{26}$, entidade filantrópica conveniada com as esferas Federal, Estadual e Municipal enquadra-se como ILPI e também proporciona o serviço do Centro Dia, oferecendo, no decorrer do dia, as refeições principais, além de todos os cuidados dos profissionais de saúde e atividades motoras, físicas e de lazer.

O total era de 49 asilados e 21 frequentadores do Centro Dia. Foram excluídos 22 moradores do Asilo devido a distúrbios cognitivos e dificuldades de audição e linguagem, assim como uso de medicamentos antidepressivos, grau avançado de Alzheimer e recusas. Dessa maneira, as entrevistas foram realizadas com 48 idosos, 27 asilados (11 mulheres e 16 homens) e 21 frequentadores do Centro Dia (16 mulheres e 5 homens).

Foram utilizados: um instrumento de coleta de dados para a caracterização sociodemográfica (sexo, raça, anos de estudo, uso da TV, quantidade de doenças e realização de atividades domésticas) e dois questionários de avaliação da QV, o WHOQOL-Bref e WHOQOL-Old, que por indicação da própria OMS, devem ser respondidos conjuntamente.

O WHOQOL-Bref consta de 26 questões, sendo uma questão para a QV global (Autoavaliação), uma para a satisfação com a saúde e 24 questões que possibilitam o cálculo de escores para representar quatro domínios: Físico, Psicológico, Relações Sociais e Meio Ambiente. Cada domínio é composto por facetas, assim o Físico engloba Dor e desconforto, Energia e fadiga, Sono e repouso, Mobilidade, Atividades da vida cotidiana, Dependência de medicação ou de tratamentos e Capacidade de trabalho; o Psicológico reúne Sentimentos positivos, Pensar/ aprender/memória/concentração, Autoestima, Imagem corporal e aparência, Sentimentos negativos e Espiritualidade/religião/crenças pessoais; as Relações Sociais aglutinam as Relações pessoais, Suporte (Apoio) social e Atividade sexual; o Meio Ambiente abrange Segurança física e proteção, Ambiente no lar, Recursos financeiros, Cuidados de saúde e sociais: disponibilidade e qualidade, Oportunidades de adquirir novas informações e habilidades, Participações e oportunidades de recreação/lazer, Ambiente físico: (poluição/ruído/ trânsito/clima) e Transporte ${ }^{27}$.

O WHOQOL-OLD constitui-se um módulo complementar de medidas genéricas da QV em adultos idosos para utilização internacional/ transcultural. Consiste em 24 itens da escala de Likert atribuídos a seis facetas: Habilidades sensoriais (funcionamento sensorial, impacto da perda de habilidades sensoriais na qualidade de vida), Autonomia (independência na velhice, capacidade ou liberdade de viver de forma autônoma e tomar decisões), Atividades Passadas, Presentes e Futuras (satisfação sobre conquistas na vida e coisas a que anseia), Participação Social (participação nas atividades cotidianas, especialmente na comunidade), Morte e Morrer (preocupações, inquietações e temores sobre a morte e morrer) e Intimidade (capacidade de ter relacionamentos pessoais e íntimos). Os escores destas seis facetas podem ser combinados para produzir um escore geral - OLD - para a QV dos idosos ${ }^{28,29}$.

Os escores de QV, para os dois questionários, são constituídos como uma escala positiva, isto é, quanto maior o escore melhor a QV, não existindo pontos de corte para se avaliar a qualidade de vida como "boa" ou "ruim".

Para a análise estatística foi utilizado o software Statiscal Package for Social Sciences (SPSS) versão $17.0^{30}$. As variáveis sociodemográficas foram analisadas por meio de frequências absoluta e relativa. Os domínios do WHOQOL-Bref e facetas do WHOQOL-Old foram analisados isoladamente e consolidados com suas respectivas sintaxes. Para a comparação dos asilados e frequentadores do Centro Dia foi utilizado o teste não paramétrico de Mann Whitney, uma vez que a normalidade da distribuição não foi verificada - teste de Kolmogorov-Smirnov. O coeficiente de correlação de Spearman foi calculado entre os domínios do WHOQOL-Bref e a QV global, assim como as facetas do WHOQOL-Old e a variável OLD. Modelos de análises de regressão linear múltipla foram construídos para verificar a contribuição dos domínios na QV global e das facetas no OLD ${ }^{31}$. Todas as análises foram realizadas ao nível de 5\% de significância. 


\section{Resultados}

O perfil sociodemográfico dos idosos participantes, segundo sexo, raça, anos de estudo, uso da TV, quantidade de doenças e realização de atividades domésticas estão representados na Tabela 1. A idade média dos homens foi de 75,4 $\pm 8,7$ anos e das mulheres 79,4 \pm 9,6 anos. Destaca-se que a variável sexo teve maior incidência de homens asilados $(59,3 \%)$ e mulheres frequentadoras do Centro Dia (76,2\%); a maioria é da raça branca $(74,1 \%$ - asilados e $81,0 \%$ - Centro Dia) e estudaram até 1 ano (51,9\% - asilados e 47,6\% - Centro Dia), destacando-se que 3 idosos possuem curso superior completo (2 asilados).

Todos os adeptos do Centro Dia assistem TV, enquanto entre os asilados 74,1\% (20) o fazem. Todos declararam não utilizar computador. A totalidade dos idosos relatou algum tipo de doença, sendo os frequentadores do Centro Dia com maior quantidade - 71,4\% (15).

Os resultados apurados pelo questionário WHOQOL-Bref (Tabela 2) apontaram que não há diferença significativa entre os domínios e a autoavaliação de QV entre os homens frequentadores do Centro Dia e os asilados, que por sua vez, apresentaram valores médios superiores a 60,0, exceto para o domínio Meio Ambiente. Já entre as mulheres verificou-se diferença estatisticamente significante nos domínios Físico e Psicológico. Também se verificaram menores valores médios dos escores em todos os domínios para as mulheres asiladas. Há considerável diferença no nível de satisfação nos domínios Físico
$(69,0 \pm 17,1$ entre as frequentadoras do Centro Dia contra 45,5 $\pm 12,3$ entre as asiladas) e Psicológico $(72,4 \pm 15,1$ nas frequentadoras do Centro Dia contra 48,1 $\pm 12,7$ nas asiladas). Os menores valores dos escores foram apurados no domínio Meio Ambiente para as duas modalidades de vínculo e sexo.

A análise do questionário de qualidade de vida, WHOQOL-Old (Tabela 3), permitiu verificar que entre os homens houve diferença estatisticamente significante nas facetas Intimidade (idosos do Centro Dia apresentaram escore de $56,3 \pm 29,3$ contra 9,4 $\pm 22,7$ dos asilados) e OLD, enquanto para as mulheres, nas facetas Autonomia, Atividades Passadas, Presentes e Futuras, Participação Social, Intimidade e OLD.

Da mesma maneira que no WHOQOL-Bref, foram verificados menores valores médios dos escores, para as mulheres asiladas, em todos os domínios. Para as mulheres sobressaíram-se os domínios Autonomia $(62,5 \pm 20,8$ para as frequentadoras do Centro Dia contra 31,3 \pm 9,3 entre as asiladas), Atividades Passadas, Presentes e Futuras $(69,5 \pm 11,6$ para as do Centro Dia contra 46,0 $\pm 15,4$ das asiladas), Participação Social (72,7 \pm 10,7 entre as frequentadoras do Centro Dia contra 44,9 $\pm 17,9$ das asiladas), Intimidade (escore médio de 38,3 \pm 31,3 entre as mulheres do Centro Dia contra 13,1 \pm 17,3 entre as asiladas) e OLD (média de 65,5 \pm 12,3 para as frequentadoras do Centro Dia contra 44,0 $\pm 10,8$ para as mulheres asiladas). A faceta Morte e Morrer apresentou os maiores valores dos escores para os dois tipos de vínculo e para ambos os sexos (Tabela 3).

Tabela 1. Frequências absoluta e relativa das variáveis sexo, raça, anos de estudo, uso da TV, quantidade de doenças e realização de atividades domésticas dos 48 idosos - 21 frequentadores do Centro Dia e 27 asilados. Bauru/SP, 2014.

\begin{tabular}{llrr}
\hline \multicolumn{1}{c}{ Variáveis } & \multicolumn{1}{c}{ Respostas } & Centro Dia & \multicolumn{1}{c}{ Asilados } \\
\hline Sexo & Masculino & $5(23,8 \%)$ & $16(59,3 \%)$ \\
& Feminino & $16(76,2 \%)$ & $11(40,7 \%)$ \\
Raça & Branca & $17(81,0 \%)$ & $20(74,1 \%)$ \\
\multirow{3}{*}{ Anos de estudo } & Preta/Parda & $4(19,0 \%)$ & $7(15,9 \%)$ \\
& Até 1 ano & $10(47,6 \%)$ & $14(51,9 \%)$ \\
& 1 a 8 anos & $10(47,6 \%)$ & $11(40,7 \%)$ \\
Uso da TV & Superior Completo & $1(4,8 \%)$ & $2(7,4 \%)$ \\
& Sim & $21(100,0 \%)$ & $20(74,1 \%)$ \\
Quantidade de doenças & Não & $0(0,0 \%)$ & $7(25,9 \%)$ \\
& Uma & $6(28,6 \%)$ & $19(70,4 \%)$ \\
Realização de atividades domésticas & $15(71,4 \%)$ & $8(29,6 \%)$ \\
& Duas ou mais & $12(57,2 \%)$ & $24(88,9 \%)$ \\
& Não & $9(42,8 \%)$ & $3(11,1 \%)$ \\
\hline
\end{tabular}


Tabela 2. Médias e desvios padrão da comparação entre os domínios apurados por meio do WHOQOL-Bref dos 21 frequentadores do Centro Dia e 27 asilados e resultado do teste estatístico. Bauru/SP, 2014.

\begin{tabular}{|c|c|c|c|c|}
\hline Domínios & Sexo & Grupos & Média \pm DP & Teste de Mann Whitney \\
\hline \multirow[t]{4}{*}{ Físico } & Masculino & Centro Dia & $63,6 \pm 16,0$ & $U=32,500$ \\
\hline & & Asilados & $67,0 \pm 14,4$ & \\
\hline & Feminino & Centro Dia & $69,0 \pm 17,1$ & $U=23,000^{* *}$ \\
\hline & & Asilados & $45,5 \pm 12,3$ & \\
\hline \multirow[t]{4}{*}{ Psicológico } & Masculino & Centro Dia & $65,8 \pm 18,0$ & $U=28,000$ \\
\hline & & Asilados & $60,4 \pm 13,8$ & \\
\hline & Feminino & Centro Dia & $72,4 \pm 15,1$ & $U=21,500^{* *}$ \\
\hline & & Asilados & $48,1 \pm 12,7$ & \\
\hline \multirow[t]{4}{*}{ Relações Sociais } & Masculino & Centro Dia & $68,3 \pm 10,9$ & $U=29,500$ \\
\hline & & Asilados & $60,9 \pm 15,4$ & \\
\hline & Feminino & Centro Dia & $74,0 \pm 13,6$ & $U=58,500$ \\
\hline & & Asilados & $62,1 \pm 22,5$ & \\
\hline \multirow[t]{4}{*}{ Meio Ambiente } & Masculino & Centro Dia & $44,4 \pm 9,7$ & $U=37,000$ \\
\hline & & Asilados & $45,3 \pm 10,9$ & \\
\hline & Feminino & Centro Dia & $51,0 \pm 10,5$ & $U=49,500$ \\
\hline & & Asilados & $42,6 \pm 10,7$ & \\
\hline \multirow[t]{4}{*}{ Autoavaliação } & Masculino & Centro Dia & $70,0 \pm 19,0$ & $U=36,500$ \\
\hline & & Asilados & $73,4 \pm 23,2$ & \\
\hline & Feminino & Centro Dia & $70,3 \pm 17,0$ & $U=74,500$ \\
\hline & & Asilados & $67,0 \pm 11,6$ & \\
\hline
\end{tabular}

${ }^{\star} \mathrm{p}<0,05 ;{ }^{* *} \mathrm{p}<0,01$.

A correlação de Spearman entre os domínios do WHOQOL-Bref (Físico, Psicológico, Relações Sociais e Meio Ambiente) e a QV global, considerando o total de idosos, apontou que todos os domínios se correlacionaram positiva e significativamente com o domínio global, apesar de as correlações serem de baixa magnitude. Assim, procedeu-se à análise de regressão linear múltipla para se verificar a contribuição de cada domínio na QV global (variável dependente). Os quatro domínios juntos explicam 40,5\% da QV global. O domínio Relações Sociais tem a menor contribuição (6,0\%), seguido do Psicológico (7,5\%) e do Meio Ambiente (9,9\%), não apresentando significância estatística. O domínio que mais impacta na QV global é o Físico (17,1\%), com diferença estatisticamente significante (Tabela 4).

$\mathrm{Na}$ Tabela 5 estão representadas as estimativas dos coeficientes da correlação de Spearman entre as facetas do WHOQOL-Old (Habilidades Sensoriais, Autonomia, Atividades Passadas, Presentes e Futuras, Participação Social, Morte e Morrer e Intimidade) e o escore geral OLD, considerando os 48 idosos, demonstrando que todas as facetas se correlacionaram positivamente e de maneira significativa com o OLD. Dessa maneira, realizou-se a análise de regressão linear múltipla para se verificar a contribuição de cada faceta no OLD (variável dependente). As 6 facetas juntas explicam 100,0\% do OLD. A faceta Participação Social foi a que mais contribuiu para o escore geral (25,3\%), seguida de Autonomia (23,6\%), Atividades Passadas, Presentes e Futuras $(21,1 \%)$, Intimidade (17,2\%), Morte e Morrer (7,8\%) e Habilidades Sensoriais (5,0\%), com diferença estatisticamente significante para todas.

\section{Discussão}

Os indivíduos que tiveram seu perfil estudado por meio de variáveis sociodemográficas, assim como a sua $\mathrm{QV}$ avaliada pelos questionários WHOQOL-Bref e WHOQOL-Old, são idosos asilados e frequentadores da modalidade Centro Dia da Instituição Vila Vicentina de Bauru/SP.

Os idosos do Centro Dia, independente do sexo, possuem maiores índices de QV em comparação aos asilados, pois apresentam uma vida e um espectro social mais amplo, maior número de atividades diárias e maior autonomia para executá-las contribuindo para uma autoestima mais elevada e até mesmo para um bom nível de saúde, o que foi confirmado por outros es- 
Tabela 3. Médias e desvios padrão da comparação entre as facetas apuradas por meio do WHOQOL-Old dos 21 frequentadores do Centro Dia e 27 asilados e resultado do teste estatístico. Bauru/SP, 2014.

\begin{tabular}{|c|c|c|c|c|}
\hline Domínios & Sexo & Grupos & Média \pm DP & Teste de Mann Whitney \\
\hline \multirow[t]{4}{*}{ Habilidades sensoriais } & Masculino & Centro Dia & $62,5 \pm 33,4$ & $U=35,500$ \\
\hline & & Asilados & $73,4 \pm 27,7$ & \\
\hline & Feminino & Centro Dia & $66,4 \pm 27,4$ & $U=72,000$ \\
\hline & & Asilados & $57,4 \pm 17,2$ & \\
\hline \multirow[t]{4}{*}{ Autonomia } & Masculino & Centro Dia & $62,5 \pm 26,9$ & $U=19,500$ \\
\hline & & Asilados & $42,6 \pm 14,7$ & \\
\hline & Feminino & Centro Dia & $62,5 \pm 20,8$ & $U=19,500^{\star *}$ \\
\hline & & Asilados & $31,25 \pm 9,3$ & \\
\hline \multirow{4}{*}{$\begin{array}{l}\text { Atividades Passadas, } \\
\text { Presentes e Futuras }\end{array}$} & Masculino & Centro Dia & $66,3 \pm 16,3$ & $U=22,000$ \\
\hline & & Asilados & $53,5 \pm 14,8$ & \\
\hline & Feminino & Centro Dia & $69,5 \pm 11,6$ & $U=17,000^{* *}$ \\
\hline & & Asilados & $46,0 \pm 15,4$ & \\
\hline \multirow[t]{4}{*}{ Participação Social } & Masculino & Centro Dia & $65,0 \pm 13,7$ & $U=24,000$ \\
\hline & & Asilados & $57,0 \pm 16,1$ & \\
\hline & Feminino & Centro Dia & $72,7 \pm 10,7$ & $U=17,500^{\star *}$ \\
\hline & & Asilados & $44,9 \pm 17,9$ & \\
\hline \multirow[t]{4}{*}{ Morte e Morrer } & Masculino & Centro Dia & $80,0 \pm 32,6$ & $U=32,000$ \\
\hline & & Asilados & $77,0 \pm 26,8$ & \\
\hline & Feminino & Centro Dia & $83,6 \pm 22,0$ & $U=72,000$ \\
\hline & & Asilados & $71,6 \pm 34,2$ & \\
\hline \multirow[t]{4}{*}{ Intimidade } & Masculino & Centro Dia & $56,3 \pm 29,3$ & $U=5,500^{* *}$ \\
\hline & & Asilados & $9,4 \pm 22,7$ & \\
\hline & Feminino & Centro Dia & $38,3 \pm 31,3$ & $U=46,000^{*}$ \\
\hline & & Asilados & $13,1 \pm 17,3$ & \\
\hline \multirow[t]{4}{*}{ OLD } & Masculino & Centro Dia & $65,4 \pm 9,2$ & $U=12,000^{*}$ \\
\hline & & Asilados & $52,2 \pm 12,4$ & \\
\hline & Feminino & Centro Dia & $65,5 \pm 12,3$ & $U=16,000^{* *}$ \\
\hline & & Asilados & $44,0 \pm 10,8$ & \\
\hline
\end{tabular}

${ }^{\star} \mathrm{p}<0,05 ;{ }^{* *} \mathrm{p}<0,01$.

Tabela 4. Estimativas dos coeficientes de correlação de Spearman e da análise de regressão linear entre os domínios do WHOQOL-Bref e a qualidade de vida global dos 48 idosos. Bauru/SP, 2014.

\begin{tabular}{|c|c|c|c|}
\hline \multirow{3}{*}{ Domínios } & \multicolumn{3}{|c|}{$\begin{array}{c}\text { Qualidade de vida global - } \\
\text { Autoavaliação }\end{array}$} \\
\hline & \multirow{2}{*}{ Correlação } & \multicolumn{2}{|c|}{ Regressão } \\
\hline & & $\mathbf{R}^{2}(\%)$ & $\beta$ \\
\hline & & & $14,360^{\#}$ \\
\hline Físico & $0,511^{\star *}$ & 17,1 & $0,356^{\star}$ \\
\hline Meio Ambiente & $0,495^{\star *}$ & 9,9 & 0,332 \\
\hline Psicológico & $0,552^{\star *}$ & 7,5 & 0,161 \\
\hline Relações Sociais & $0,304^{*}$ & 6,0 & 0,129 \\
\hline $\mathrm{R}^{2}$ Total (\%) & & 40,5 & \\
\hline
\end{tabular}

tudos ${ }^{13,23-25}$. Um dos aspectos importantes para melhor percepção da QV é o convívio social dos idosos, praticado no Centro Dia, onde são estabelecidas ações que promovem a formação de grupos para a realização de atividades físicas, lazer, culturais e de trabalho, diferente dos asilados, que passam o dia todo na Instituição, têm uma rotina geralmente monótona e de baixa autonomia nas atividades, convívio social limitado, com, geralmente, poucas visitas de familiares e amigos. Isso contribui para baixa autoestima e valorização da própria QV.

Além destas, percebem-se diferenças nas apurações do que os indivíduos consideram QV entre mulheres e homens, retratados nos melhores escores da QV entre os homens. Isto se deve também, em aspectos gerais, à compreensão diferente, entre os sexos, do que é a velhice. As mulheres costumam sentir muito mais a chegada 
Tabela 5. Estimativas dos coeficientes de correlação de Spearman e da análise de regressão linear entre as facetas do WHOQOL-Old e a qualidade de vida global dos 48 idosos. Bauru/SP, 2014.

\begin{tabular}{|c|c|c|c|}
\hline \multirow{3}{*}{ Facetas } & \multicolumn{3}{|c|}{ Escore geral - OLD } \\
\hline & \multirow{2}{*}{ Correlação Spearman } & \multicolumn{2}{|c|}{ Regressão } \\
\hline & & $\mathbf{R}^{2}(\%)$ & $\beta$ \\
\hline & & & $-2,309 \mathrm{E}-14^{\#}$ \\
\hline Participação Social & $0,861^{\star *}$ & 25,3 & $0,167^{\star *}$ \\
\hline Autonomia & $0,721^{\star *}$ & 23,6 & $0,167^{\star *}$ \\
\hline Atividades Passadas, Presentes e Futuras & $0,784^{\star *}$ & 21,1 & $0,167^{\star *}$ \\
\hline Intimidade & $0,694^{* *}$ & 17,2 & $0,167^{\star *}$ \\
\hline Morte e Morrer & $0,463^{\star *}$ & 7,8 & $0,167^{\star *}$ \\
\hline Habilidades Sensoriais & $0,361^{*}$ & 5,0 & $0,167^{* *}$ \\
\hline $\mathrm{R}^{2}$ Total $(\%)$ & & 100,0 & \\
\hline
\end{tabular}

\#Valor da constante; ${ }^{*} \mathrm{p}<0,05 ;{ }^{* *} \mathrm{p}<0,01$.

da idade e se sentem mais incomodadas com ela, entendendo-a como algo negativo e relacionado a problemas e limitações, dependência, finitude, feiura e medo ${ }^{15,18,26,32}$. Já os homens, entendem a velhice como fenômeno universal, significando aposentadoria, dependência e doença, portanto acostumam-se mais rápido com as fragilidades, além de estarem menos expostos aos problemas físicos e mentais. Na pesquisa realizada em Minas Gerais $^{32}$, com 77 idosos institucionalizados, também ficou evidente os maiores escores para os homens em todos os domínios da QV em relação às mulheres, assim como no Rio Grande do Sul, onde 364 idosos foram entrevistados ${ }^{18} \mathrm{e}$ na Paraíba junto a 69 integrantes de quatro instituições asilares de João Pessoa ${ }^{19}$.

Os idosos apresentaram baixo nível de estudo, ou seja, 50\% tem menos de 1 ano, podendo ser considerados analfabetos funcionais ${ }^{2}$, revelando a cultura de vida e da época em que estes idosos tinham como contexto de estudo quando jovens. Muitos paravam de estudar cedo para ajudar nas atividades de trabalho e no sustento da casa no caso dos homens, e na pouca importância dada à época para a educação das mulheres. De acordo com o IBGE ${ }^{2}$, uma das características marcantes da população idosa brasileira é seu baixo nível de escolaridade, tendo em vista que cerca de $25 \%$ da população idosa brasileira é de analfabetos. Pode-se inferir que, geralmente, quanto maior o grau de educação, maior a noção do mundo que há a sua volta e da capacidade de avaliação própria do nível da sua QV, como identificado em outros estudos ${ }^{13,15-17,23,24}$.

Observou-se que os idosos praticam poucas atividades domésticas, mesmo os do Centro Dia, fazendo com que se sintam menos capazes, po- dendo tornar-se mais tristes e descontentes com sua situação, também influenciando na baixa avaliação da própria QV. Algumas pesquisas também apontam o alto comportamento sedentário que caracteriza a população idosa, motivado pelos prejuízos nos sistema estrutural e fisiológico advindos do envelhecimento, assim como da própria institucionalização, levando-os à incapacidade funcional ${ }^{16,18,32-34}$.

Os resultados do questionário WHOQOL -Bref permitiram constatar que as usuárias do Centro Dia apresentaram maiores escores em relação às asiladas em todos os domínios, destacando-se grandes diferenças entre os escores médios nos domínios Físico e Psicológico.

Ao se analisar a contribuição dos diferentes domínios na QV global, observou-se que a contribuição dos quatro domínios juntos foi de $40,5 \%$, e que os domínios diferiram a respeito da contribuição individual na QV global, sendo que o domínio que mais contribuiu foi o Físico (17,1\%), seguido do Meio Ambiente (9,9\%), Psicológico (7,5\%) e Relações Sociais $(6,0 \%)$, embora os 3 últimos não tenham apresentado diferenças estatisticamente significantes.

A capacidade funcional, autonomia e independência, avaliadas no domínio Físico, constituem-se importantes fatores de impacto na QV em idosos, confirmados pela regressão linear, com diferença expressiva para as mulheres, fato que pode estar relacionado ao receio em depender de outras pessoas na idade adulta. Resultado semelhante foi apontado em estudo realizado no Rio Grande do Sul entre idosos com incapacidade funcional, que evidenciou 36,1 vezes mais risco de apresentar pior QV no domínio Físico do que os não possuíam incapacidades ${ }^{35}$. 
As idosas de hoje, geralmente, são aquelas que, antes da idade avançada, tinham uma rotina de muita importância familiar e profissional, assim, quando passam a morar na Instituição, sentem falta de seus familiares, amigos e das atividades domésticas, com as quais estavam acostumadas, $\mathrm{e}$ que não possuem mais capacidade física ou mental para realizar, além dos fatores esquecimento e o sentimento de que mulheres mais velhas são menos atraentes ${ }^{15,18,36}$. Dessa maneira, compreende-se o fato de que o próprio deslocamento diário das mulheres para o Centro Dia permite que sintam-se física e socialmente mais ativas.

No aspecto Psicológico foram revelados valores médios dos domínios muito próximos entre os asilados e os do Centro Dia e acima de 60,4 para os homens, ou seja, a institucionalização não influenciou a percepção da QV dos idosos, como também demonstrado no estudo com 77 sujeitos de $\mathrm{MG}^{32}$. Por outro lado, enquanto as mulheres do Centro Dia apresentaram escore médio de $72,4 \pm 15,1$, as asiladas tiveram um desempenho bem inferior - 48,1 $\pm 12,7$. A experiência do asilamento para as idosas está relacionada com o sentimento de perda de liberdade, identidade, autonomia e confiança, assim como abandono pelos filhos e aproximação da morte, intensificando os estados de solidão e dependência, influenciando diretamente o aspecto psicológico $^{19,37}$. Os estudos realizados no interior de São Paulo e em Minas Gerais contrariam tal achado, enfatizando que o convívio social e a prática de atividades em conjunto, na Instituição, levam ao bem-estar psicológico, demonstrando melhores escores em tal domínio ${ }^{38,39}$.

No domínio das Relações Sociais não foi observada diferença estatística para ambos os sexos e os valores dos escores foram todos acima de 60,9 , embora menores para os asilados. Considerando que tal domínio aglutina as relações interpessoais e o suporte social, pode-se entender que as perdas sociais a que os idosos são expostos, ou seja, papéis do cotidiano (profissional, político ou familiar), são recompensadas com o novo convívio proporcionado pelas atividades do Centro Dia e até mesmo da Instituição, não representando prejuízo à QV dos idosos, da mesma maneira que os achados nos estudos em 3 ILPI do interior de São Paulo ${ }^{21}$, em Caxias do Sul/RS ${ }^{22}$ e na região metropolitana de São Paulo ${ }^{13}$.

Segundo a OMS, o ambiente físico em que o idoso está inserido pode determinar a dependência ou não do indivíduo ${ }^{3}$. Dessa forma, é provável que um idoso esteja física e socialmente ativo se puder ir caminhando com segurança às depen- dências da Instituição, ao jardim, ou tomar sol, em um local acessível, calçado e higienizado, assim como arborizado, tranquilo, confortável e aconchegante, como é o caso da ILPI do estudo, que possui, inclusive uma capela com missas diárias. Lamentavelmente, os menores valores de escores foram apurados no domínio Meio Ambiente para as duas modalidades e sexos, sendo inferiores a 51,0, confirmando, dessa maneira, a influência dos aspetos físicos na interação com o meio ambiente. Tais resultados são contrários aos encontrados em ILPI públicas e privadas no interior do estado do Rio Grande do Sul ${ }^{39}$, com 148 idosos em Santa Rita do Sapucaí2 ${ }^{28}$, com 15 idosos participantes do grupo de convivência Nossa Senhora de Fátima ${ }^{15}$, mas estão em concordância com outras pesquisas ${ }^{23,35,40}$.

Ao analisar os resultados do WHOQOL-Old, verifica-se que o escore geral OLD foi estatisticamente significante para os dois sexos entre as modalidades de vínculo, destacando-se o menor escore $(44,0 \pm 10,8)$ para as asiladas. A análise de regressão linear múltipla permitiu verificar que as 6 facetas juntas explicam 100,0\% do OLD, sendo a Participação Social a que mais impactou $(25,3 \%)$ e Habilidades Sensoriais $(5,0 \%)$ a de menor influência no escore total.

A faceta Participação Social do WHOQOL -OLD destaca a participação em atividades da comunidade, satisfação com o nível de atividade diária e utilização do tempo. Os idosos, principalmente as mulheres, consideram de fundamental importância manter os relacionamentos interpessoais, conservar fortalecidos os vínculos com a família, contribuindo, se possível com a educação de filhos e netos, bem como estendendo a vizinhos e amigos, solidificando sua rede de suporte social ${ }^{5,8}$. Nos resultados do presente estudo, observou-se que os melhores escores nas facetas do domínio Participação Social estiveram relacionados aos frequentadores do Centro Dia de ambos os sexos.

Sem revelar diferenças estatísticas, com escores médios superiores a 57,4 e influenciando minimamente o escore OLD, a faceta Habilidades Sensoriais pode ser interpretada como uma indicação de que os idosos desse estudo, em geral, não apresentaram perdas sensoriais que possam afetar sua vida diária, assim como sua participação nas atividades e interação com as pessoas que residem na Instituição e frequentam o Centro Dia, concordando com outros estudos ${ }^{13,15,20,39}$.

A faceta Intimidade, que retrata a capacidade de ter relacionamentos pessoais e íntimos, apresentou os piores escores de $\mathrm{QV}$, inclusive 
com valores zero, com diferença significante para ambos os sexos. Para os homens asilados, tratase do menor valor apurado $(9,4)$ entre todas as facetas, embora no desempenho global da faceta, as mulheres continuam destacando-se, pois o escore médio foi superior $(13,1)$, mas os valores máximos e mínimos foram entre 0,0 e 50,0. Alguns autores enfatizam que o ato sexual em si talvez seja menos importante nesta faixa etária do que a possibilidade de intimidade, ou seja, os sentimentos de companheirismo e afeto e as oportunidades para amar e ser amado a $^{3,20,21}$. Porém, se tal faceta for analisada do ponto de vista da sexualidade, justificam-se os escores ruins, assim como em outros estudos ${ }^{36,39}$, uma vez que as características das ILPI não possibilitam espaços privados para casais ${ }^{23,41}$. Provavelmente a avaliação da intimidade para os idosos do estudo, principalmente para as mulheres asiladas, foi relativa à sexualidade, pois os escores do domínio Relações Sociais (WHOQOL-Bref) superiores a 60,9 e da faceta Participação Social acima de 57,0, indicam que não há problemas de companheirismo entre os homens e com as mulheres do Centro Dia.

Algumas diferenças importantes foram observadas na faceta Autonomia, tendo os asilados os menores valores, 42,6 e 31,3 para os homens e mulheres, respectivamente. Tal faceta enfatiza a independência na velhice, capacidade ou liberdade de viver de forma autônoma e tomar decisões, relacionando-se ao domínio Físico, já avaliado com os menores valores para as asiladas. Os idosos que se encontram em ILPI são geralmente pessoas passivas, com poucas atividades ocupacionais e iniciativas próprias para realizar algo que preencha seu tempo. Resultados semelhantes foram observados no Rio Grande do Norte ${ }^{20}$, Rio Grande do Sul ${ }^{15}$, Minas Gerais ${ }^{32}$, Piauí ${ }^{34}$, Ceará ${ }^{38}$ e São Paulo ${ }^{39}$. A dependência, física ou mental, prejudica sobremaneira a QV dos idosos, constituindo-se um fator de risco importante para mortalidade. Dessa maneira, todas as iniciativas de promoção de saúde, de assistência e de reabilitação em saúde devem ter como meta valorizar a autonomia e a independência física e mental do idoso $5,13,42$

Os resultados relativos à faceta Atividades Passadas, Presentes e Futuras também foram inferiores para os homens e mulheres asilados, o que demonstra uma falta de perspectiva de vida dos asilados, que já não projetam mais atividades e interesses futuros, devendo-se em parte à dependência física e falta de autonomia, já demonstradas anteriormente e de acordo com outras pesquisas realizadas com idosos institucionali$\operatorname{zados}^{15,20,32,42}$. Esta faceta aponta para a satisfação dos idosos quanto a realizações, objetivos alcançados e projetos durante a vida, apontando para uma boa QV em alguns estudos ${ }^{38,39,40}$.

$\mathrm{Na}$ faceta Morte e Morrer os escores médios foram acima de 77,0 e 71,6 para homens e mulheres, respectivamente, indicando que os idosos desse estudo, independente da condição de institucionalizados ou não, estão satisfeitos em relação aos sentimentos, inquietações e temores com a morte e morrer. Provavelmente, por se sentirem no final da vida, a morte signifique algo já esperado e certo para eles e tenham desenvolvido algum mecanismo de enfrentamento em relação à vida e ao futuro, destacando-se a importância da espiritualidade, fé e religiosidade. Resultados semelhantes estão de acordo com outros encontrados na literatura ${ }^{15,20,32,38,40}$.

As limitações do estudo referiram-se ao seu desenho transversal, que não permitiu relação de causa e efeito, e à retratação da realidade de uma população específica, sem a possibilidade de generalização dos dados. Apesar disso, os resultados devem contribuir para o aumento das evidências científicas relacionadas ao tema QV, envelhecimento e institucionalização, e para minimizar os riscos aos quais os idosos institucionalizados estão propensos, mediante a avaliação dos níveis de fragilidade e de QV. Os instrumentos utilizados mostraram-se sensíveis à abordagem pretendida, mas para o aprofundamento do tema estudado seria interessante realizar uma pesquisa com delineamento qualitativo por meio de entrevista semiestruturada para uma particularização dos sujeitos e melhor detalhamento dos domínios e das facetas envolvidos na determinação da QV.

\section{Considerações finais}

Os resultados permitiram verificar melhores escores médios de QV para os idosos do Centro Dia, onde se destacaram as mulheres. Entre os institucionalizados, as mulheres tiveram os piores valores de QV. Os domínios com escores mais baixos foram Meio Ambiente e Intimidade, enquanto os mais altos foram Relações Sociais e Morte e Morrer para os dois tipos de vínculo com a Instituição e ambos os sexos.

Os domínios que mais contribuíram para os escores totais de QV foram o Físico (apurado no WHOQOL-Bref) e a Participação Social (WHOQOL-OLD). A maior influência do domínio Físico na QV global ressalta a importância de promover 
atividades que estimulem a autonomia e independência, visando à melhoria da capacidade funcional e, consequentemente, da QV. Tais atividades também reforçariam as relações interpessoais e o suporte social, avaliadas no domínio Participação Social, pois um dos aspectos importantes para melhor percepção da QV é o convívio social dos idosos, que se dá no Centro Dia e na Instituição.

A utilização dos instrumentos WHOQOL -Bref e WHOQOL-Old foram excelentes indicadores da real situação dos idosos e se complementaram nas análises constituindo-se instrumentos válidos e fiéis para a avaliação da QV genérica de idosos asilados e frequentadores do Centro Dia, inclusive levando em consideração as especificidades do envelhecimento humano.
Considera-se que a institucionalização não proporciona piora na QV da pessoa idosa e sim, que a percepção dessa qualidade pode já estar comprometida quando se procura a institucionalização. Por outro lado, a assistência proporcionada no Centro Dia potencializou melhora ou manutenção da QV dos participantes, revelando ser uma alternativa promissora de modalidade de serviço para a saúde do idoso. Tendo em vista a variabilidade do conceito de QV e sua subjetividade, com o propósito de se orientar as políticas para um envelhecimento bem sucedido, parece imprescindível conhecer o que, para a maioria dos idosos, está relacionado ao bem-estar, à felicidade, à realização pessoal, enfim, à QV nessa faixa etária.

\section{Colaboradores}

SFAP Simeão: Concepção e desenho do estudo, Análise e interpretação dos dados. GAL Martins: Concepção e desenho do estudo e coleta de dados. MANGatti:Interpretação dos dados erevisãofinal. MHS De Conti e SN Marta: Revisão final. A De Vitta: Análise e interpretação dos dados, revisão final. 


\section{Referências}

1. Organización Panamericana de la Salud (OPAS). Guia Clínica para Atención Primaria a las Personas Mayores. $3^{a}$ ed. Washington: OPAS; 2003.

2. Instituto Brasileiro de Geografia e Estatística (IBGE). Estatísticas sobre a população 2013. 2013. [acessado 2014 Fev 18]. Disponível em: http://www.ibge.gov.br

3. Organização Mundial da Saúde (OMS). Índice de desenvolvimento humano. Programa das nações unidas para o desenvolvimento. [relatório]. 2010. [acessado 2014 Mar 3]. Disponível em: http://www.pnud.org.br

4. The WHOQOL Group. Mensuração da qualidade de vida (WHOQOL). Geneve: WHO; 1993.

5. Vecchia RD, Ruiz T, Bocchi SCM, Corrente JE. Qualidade de vida na terceira idade: um conceito subjetivo. Rev Bras Epidemiol 2005; 8(3):246-252.

6. Velarde JE, Avila FC. Methods for quality of life assessment. Salud Pública Méx 2002; 44(4):349-361.

7. Bowling A, Gabriel Z, Dakes J, Dowding LM, Evans O, Fleissig A, Banister D, Sutton S. Let's ask them: a national survey of definitions of quality of life and its enhancement among people aged 65 and over. Int J Aging Hum Dev 2003; 56(4):269-306.

8. Vagetti GC, Moreira NB, Barbosa Filho VC, Oliveira V, Cancian CF, Mazzardo O, Campos W. Domínios da qualidade de vida associados à percepção de saúde: um estudo com idosas de um programa de atividade física em bairros de baixa renda de Curitiba, Paraná, Brasil. Cien Saude Colet 2013; 18(12):3483-3493.

9. Campos MRX, Dias CA, Rodrigues SM. Representações de cuidadores de idosos a respeito do "ser idoso", da "velhice" e do "viver institucionalizado". Rev Bras Cien Envelhecimento Humano 2011; 8(2):255-264.

10. Perlini NMOG, Leite MT, Furini AC. Em busca de uma instituição para a pessoa idosa morar: motivos apontados por familiares. Rev esc enferm USP 2007;41(2):231234.

11. Brasil. Portaria no 810 , de 22 de setembro de 1989, que dispõe sobre as normas para o funcionamento de casa de repouso, clínicas geriátricas e outras instituições destinadas ao atendimento de idosos. Diário Oficial da União 1989; 22 set.

12. Brasil. Portaria do Gabinete do Ministro de Estado da Saúde de no 1395, de 9 de dezembro de 1999, que aprova a Política Nacional de Saúde do Idoso e dá outras providências. Diário Oficial da União 1999; $13 \mathrm{dez}$.

13. Franciulli SE, Ricci NA, Lemos ND, Cordeiro RC, Gazzola JM. A modalidade de assistência Centro Dia Geriátrico: efeitos funcionais em seis meses de acompanhamento multiprofissional. Cien Saude Colet 2007; 12(2):373-380.

14. Black DA. The geriatric day hospital. Age Ageing 2005; 34(5):427-429.

15. Serbim AK, Figueiredo AEPL. Qualidade de vida de idosos em um grupo de convivência. Scientia Medica 2011; 21(4):166-172.

16. Oliveira MPF, Novaes MRCG. Perfil socioeconômico, epidemiológico e farmacoterapêutico de idosos institucionalizados de Brasília, Brasil. Cien Saude Colet 2013; 18(4):1069-1078.

17. Danilow MZ, Moreira ACS, Villela CG, Barra BB, Novaes MRCG, Oliveira MPF. Perfil epidemiológico e psicossocial de idosos institucionalizados do Distrito Federal. Comun Ciênc Saúde 2007; 18(1):9-16.
18. Vitorino LM, Paskulin LMG, Vianna LAC. Quality of life of seniors living in the community and in long term care facilities: a comparative study. Rev Latino-Am. Enfermagem 2013; 21(Spec):3-11.

19. Lima DL, Lima MAVD, Ribeiro CG. Envelhecimento e qualidade de vida de idosos institucionalizados. Rev Bras Cien Envelhecimento Humano 2010; 7(3):346-356.

20. Nunes VMA, Menezes RMP, Alchieri JC. Avaliação da Qualidade de Vida em idosos institucionalizados no município de Natal, Estado do Rio Grande do Norte. Acta Scientiarum: Health Science 2010; 32(2):119-126.

21. Freitas MAV, Scheicher ME. Qualidade de vida de idosos institucionalizados. Rev Bras Geriatr Gerontol 2010; 13(3):395-402.

22. Mincato PC, Freitas CLR. Qualidade de vida dos idosos residentes em instituições asilares da cidade de Caxias do Sul-RS. Rev Bras Cien Envelhecimento Humano 2007; 4(1):127-138.

23. Sagalla R, Spinelli RB, Zanardo VPS, Zemolin GP. Perfil antropométrico e qualidade de vida de idosos independentes institucionalizados e não institucionalizados, no município de Erechim, RS. Revista Perspectiva 2013; 37(137):81-92.

24. Khoury HTT, Sá-Neves AC. Percepção de controle e qualidade de vida: comparação entre idosos institucionalizados e não institucionalizados. Rev Bras Geriatr Gerontol 2014; 17(3):553-565.

25. Moreira PA, Roriz AKC, Mello AL, Ramos LB. Quality of Life of Institutionalized Elderly in Brazil. Soc Indic Res 2016; 126:187-197.

26. Araujo GS, Gatti MAN, De Conti MHS, De Vitta A, Marta SN, Simeão SFAP. Qualidade de vida de idosos residentes na Vila Vicentina de Bausu/SP. Salusvita 2014; 33(1):57-75.

27. Fleck MPA, Louzada S, Xavier M, Chachamovich E, Vieira G, Santos L, Pinzon V. Aplicação da versão em português do instrumento abreviado de avaliação da qualidade de vida “WHOQOL-Bref”. Rev Saude Publica 2000; 34(2):178-183.

28. Power M, Quinn K, Schmidt S, The WHOQOL-OLD Group. Development of the WHOQOL-OLD module. Qual Life Res 2005; 14(10):2197-2214.

29. Fleck MPA, Chachamovich E, Trentini C. Development and validation of the Portuguese version of the WHOQOL-OLD module. Rev Saude Publica 2006; 40(5):785-791.

30. SPSS Inc. SPSS Statistics 17.0 Brief Guide. Chicago: SPSS Inc.; 2007.

31. Zar JH. Biostatistical Analysis. $5^{\text {th }}$ ed. New Jersey: Prentice-Hall; 2010.

32. Vitorino LM, Paskulin LMG, Vianna LAC. Quality of life among older adults resident in long-stay care facilities. Rev Latino-Am. Enfermagem 2012; 20(6):11861195.

33. Tavares DMS, Dias FA. Capacidade funcional, morbidades e qualidade de vida de Idosos. Texto Contexto Enfermagem 2012; 21(1):112-20.

34. Sousa SS, Oliveira PS, Oliveira FS, Holanda MAG, Almeida PC, Machado ALG. Study of sociodemographic factors associated with functional dependence in the elderly. Rev Enf Universidade Federal do Piauí 2013; 2(1):44-48. 
35. Pereira RJ, Cotta RMM, Franceschini SCC, Ribeiro RCL, Sampaio RF, Priore SE, Cecon PR. Contribuição dos domínios físico, social, psicológico e ambiental para a qualidade de vida global de idosos. Rev Psiquiatr Rio Gd Sul 2006; 28(1):27-38.

36. Avis NE, Assmann SF, Kravitz HM, Ganz PA, Ory M. Quality of life in diverse groups of midlife women: assessing the influence of menopause, health status and psychosocial and demographic factors. Qual Life Res 2004; 13(5):933-946.

37. Cordeiro LM, Paulino JL, Bessa MEP, Borges CL, Leite SFP. Quality of life of frail and institutionalized elderly. Acta Paul Enferm 2015; 28(4):361-366.

38. Dias DSG, Carvalho CS, Araújo CV. Comparação da percepção subjetiva de qualidade de vida e bem-estar de idosos que vivem sozinhos, com a família e institucionalizados. Rev Bras Geriatr Gerontol 2013; 16(1):127-138.

39. Freire Junior RC, Tavares MFL. A saúde sob o olhar do idoso institucionalizado: conhecendo e valorizando sua opinião. Interface (Botucatu) 2005; 9(16):147-158.

40. Binelo J, Mattjie ML, Ilha VD. A qualidade de vida de idosos institucionalizados. Revista de Psicologia da IMED 2015; 7(1):35-41.

41. Mahieu L, Gastmans C. Sexuality in institutionalized elderly persons: a systematic review of argument-based ethics literature. Int Psychogeriatr 2012; 24(3):346-357.

42. Paskulin LMG, Cordova FP, Costa FM, Vianna LAC. Percepção de pessoas idosas sobre qualidade de vida. Acta paul. enferm 2010; 23(1):101-107.

Artigo apresentado em 20/04/2016

Aprovado em 23/11/2016

Versão final apresentada em 25/11/2016 